\title{
Research Article \\ Finite-Time Attractivity for Diagonally Dominant Systems with Off-Diagonal Delays
}

\author{
T. S. Doan ${ }^{1,2}$ and S. Siegmund ${ }^{3}$ \\ ${ }^{1}$ Department of Mathematics, Imperial College London, London SW7 2AZ, UK \\ ${ }^{2}$ Institute of Mathematics, Vietnam Academy of Science and Technology, Hanoi 10307, Vietnam \\ ${ }^{3}$ Center for Dynamics, TU Dresden, 01062 Dresden, Germany
}

Correspondence should be addressed to S. Siegmund, siegmund@tu-dresden.de

Received 23 April 2012; Accepted 6 June 2012

Academic Editor: Agacik Zafer

Copyright (c) 2012 T. S. Doan and S. Siegmund. This is an open access article distributed under the Creative Commons Attribution License, which permits unrestricted use, distribution, and reproduction in any medium, provided the original work is properly cited.

We introduce a notion of attractivity for delay equations which are defined on bounded time intervals. Our main result shows that linear delay equations are finite-time attractive, provided that the delay is only in the coupling terms between different components, and the system is diagonally dominant. We apply this result to a nonlinear Lotka-Volterra system and show that the delay is harmless and does not destroy finite-time attractivity.

\section{Introduction}

Finite-time dynamical systems generated by nonautonomous differential equations which are defined only on a compact interval of time have recently become an active field of research, see, for example, [1-3] and the references therein.

A key ingredient of a qualitative theory is the notion of hyperbolicity of solutions. Finite-time versions of hyperbolicity are introduced and discussed, for example, in [4-8]. Finite-time attractivity is a special case of finite-time hyperbolicity, in case the unstable direction is missing. So far finite-time attractivity has been discussed only for ordinary differential equations, see $[9,10]$. For a closely related notion, namely, finite-time stability, we refer to $[11,12]$ and the references therein for an overview. In this paper we go one step further to extend and investigate finite-time attractivity for delay equations.

For a nonnegative number $r \geq 0$, let $C:=C\left([-r, 0], \mathbb{R}^{d}\right)$ denote the space of all continuous functions $\varphi:[-r, 0] \rightarrow \mathbb{R}^{d}$. For $\gamma \in \mathbb{R}$, the norm $\|\cdot\|_{\gamma, \infty}$ on $\mathcal{C}$ is defined as follows:

$$
\|\varphi\|_{r, \infty}:=\max \left\{e^{r s}\|\varphi(s)\|_{\infty}: s \in[-r, 0]\right\},
$$


where $\|x\|_{\infty}=\max \left\{\left|x_{i}\right|: i=1, \ldots, d\right\}$ for all $x=\left(x_{1}, \ldots, x_{d}\right)^{T} \in \mathbb{R}^{d}$. Consider a finite-time delay differential equation

$$
\dot{x}=f\left(t, x_{t}\right) \text { for } t \in[0, T],
$$

where $f:[0, T] \times \mathcal{C} \rightarrow \mathbb{R}^{d}$ is assumed to be continuous and Lipschitz in the second argument. For each $\varphi \in \mathcal{C}$, let $x(\cdot, \varphi)$ denote the solution of $(2.1)$ satisfying the initial condition $x(s)=$ $\varphi(s)$ for all $s \in[-r, 0]$. The evolution operator $S:[0, T] \times \mathcal{C} \rightarrow \mathcal{C}$ generated by (1.2) is defined as

$$
(S(t) \varphi)(s)=x(t+s, \varphi) \quad \forall s \in[-r, 0], t \in[0, T] .
$$

Motivated by recent results on finite-time hyperbolicity (see, e.g., $[4,6,7]$ ), we introduce in the following an analog notion of finite-time attractivity for delay equations.

Definition 1.1 (finite-time attractivity). The solution $S(\cdot, \varphi)$ is called finite-time attractive on $[0, T]$ with respect to the norm $\|\cdot\|_{\gamma, \infty}$ if there exist positive constants $\alpha$ and $\eta$ such that for all $t, s \in[0, T]$ with $s \leq t$ the following estimate holds:

$$
\|S(t, \varphi)-S(t, \psi)\|_{\gamma, \infty} \leq e^{-\alpha(t-s)}\|S(s, \varphi)-S(s, \psi)\|_{\gamma, \infty}
$$

for all $\psi$ in the neighborhood $B_{\eta}(\varphi)$ of $\varphi$.

Remark 1.2. In the case that $f:[0, T] \times \mathcal{C} \rightarrow \mathbb{R}^{d}$ is a linear function in the second argument, it is easy to see that the generated semigroup $S:[0, T] \times \mathcal{C} \rightarrow \mathcal{C}$ is also linear in the second argument. In particular, for linear systems the following statements are equivalent:

(i) there exists a finite-time attractive solution $S(\cdot, \varphi)$ for a $\varphi \in \mathcal{C}$,

(ii) for all $\varphi \in \mathcal{C}$, the solution $S(\cdot, \varphi)$ is finite-time attractive, and

(iii) there exists $\alpha>0$ such that for all $0 \leq s \leq t \leq T$ we have

$$
\|S(t, \varphi)\|_{\gamma, \infty} \leq e^{-\alpha(t-s)}\|S(s, \varphi)\|_{\gamma, \infty} \quad \forall \varphi \in \mathcal{C} .
$$

In this paper, we prove the finite-time attractivity for linear off-diagonal delay systems in Section 2. Section 3 is devoted to show the finite-time attractivity of the equilibrium for a Lotka-Voltera system.

\section{Finite-Time Attractivity for Linear Off-Diagonal Delay Systems}

In this section, we consider the following finite-time nonautonomous linear differential equation with off-diagonal delays (see, e.g., [13] and the reference therein):

$$
\dot{x}_{i}(t)=a_{i i}(t) x_{i}(t)+\sum_{j=1, j \neq i}^{d} a_{i j}(t) x_{j}\left(t-\tau_{i j}\right) \quad \text { for } i=1, \ldots, d, t \in[0, T]
$$


where $T$ is a given positive constant, $a_{i j}: \mathbb{R} \rightarrow \mathbb{R}, i, j=1, \ldots, d$, are continuous functions and $\tau_{i j}>0$ for $i, j=1, \ldots, d$ with $i \neq j$. Define

$$
r:=\max \left\{\tau_{i j}: i, j=1, \ldots, d, i \neq j\right\} .
$$

Note that (2.1) is a special case of (1.2). More precisely, the right hand side of (2.1) equals $f\left(t, x_{t}\right)$, where $f=\left(f_{1}, \ldots, f_{d}\right):[0, T] \times \mathcal{C} \rightarrow \mathbb{R}^{d}$ is defined as follows:

$$
f_{i}(t, \varphi):=a_{i i}(t) \varphi(0)+\sum_{j=1, j \neq i}^{d} a_{i j}(t) \varphi_{j}\left(-\tau_{i j}\right) .
$$

Let $S:[0, T] \times \mathcal{C} \rightarrow \mathcal{C}$ denote the evolution operator of (2.1). From (2.3), we see that the function $f$ is linear in the second argument. Therefore, the evolution operator $S$ is also linear in the second argument. Our aim in this section is to provide a sufficient condition for the finite-time attractivity for the zero solution of (2.1) and thus for all solutions of (2.1), see Remark 1.2.

Before presenting the main result, we recall the notion of row diagonal dominance. We refer the reader to [14, Definition 7.10] for a discussion of this notion. System (2.1) is called row diagonally dominant if there exists a positive constant $\delta$ such that

$$
\left|a_{i i}(t)\right| \geq \sum_{j=1, j \neq i}^{d}\left|a_{i j}(t)\right|+\delta \quad \text { for } t \in[0, T] .
$$

Theorem 2.1 (finite-time attractivity for delay equations). Consider system (2.1) on a finite-time interval $[0, T]$. Suppose that system (2.1) is row diagonally dominant with a positive constant $\delta$ and $a_{i i}(t)<0$ for all $i=1, \ldots, d$ and $t \in[0, T]$. Define

$$
M:=\max \left\{\sum_{j=1, j \neq i}^{d}\left|a_{i j}(t)\right|: i=1, \ldots, d, t \in[0, T]\right\},
$$

and let $\gamma^{*}$ be a positive number satisfying that

$$
\frac{r^{*}}{2}+\left(e^{r^{*} r}-1\right) M=\delta
$$

Then for every $\gamma \in\left[0, \gamma^{*}\right]$, the zero solution of (2.1) is finite-time attractive with respect to the norm $\|\cdot\|_{\gamma, \infty}$ with exponent $-\gamma / 2$, that is,

$$
\|S(t, \varphi)\|_{\gamma, \infty} \leq e^{-(\gamma / 2)(t-s)}\|S(s, \varphi)\|_{\gamma, \infty} \text { for } 0 \leq s \leq t \leq T .
$$

Proof. We divide the proof into two steps.

Step 1 . We show that for $\varphi \in \mathcal{C}$ the inequality

$$
\|x(t, \varphi)\|_{\infty} \leq e^{-(\gamma / 2)(t-s)}\|S(s, \varphi)\|_{\gamma, \infty} \quad \forall 0 \leq s \leq t \leq T
$$


holds. Suppose the opposite, that is, assume that there exists $s \in[0, T]$ such that the set

$$
\mathcal{N}:=\left\{t \in[s, T):\|x(t, \varphi)\|_{\infty}>e^{-(\gamma / 2)(t-s)}\|S(s, \varphi)\|_{\gamma, \infty}\right\}
$$

is not empty. Define $t_{\text {inf }}=\inf \{t: t \in \mathcal{N}\}$. By continuity of the map $t \mapsto\|x(t, \varphi)\|_{\infty}$, we get that

$$
\begin{gathered}
\left\|x\left(t_{\mathrm{inf}}, \varphi\right)\right\|_{\infty}=e^{-(\gamma / 2)\left(t_{\mathrm{inf}}-s\right)}\|S(s, \varphi)\|_{\gamma, \infty^{\prime}} \\
\|x(t, \varphi)\|_{\infty} \leq e^{-(\gamma / 2)(t-s)}\|S(s, \varphi)\|_{\gamma, \infty} \quad \forall t \in\left[s, t_{\mathrm{inf}}\right] .
\end{gathered}
$$

Now, we will show that

$$
\left\|x\left(t_{\text {inf }}, \varphi\right)\right\|_{\infty} \geq e^{-\gamma r}\|x(t, \varphi)\|_{\infty} \quad \forall t \in\left[t_{\text {inf }}-r, t_{\text {inf }}\right] .
$$

Indeed, we consider the following two cases: (i) $t \in\left[s, t_{\text {inf }}\right] \cap\left[t_{\text {inf }}-r, t_{\text {inf }}\right]$ and (ii) $t \in(-\infty, s] \cap$ $\left[t_{\text {inf }}-r, t_{\text {inf }}\right]$.

Case (i). If $t \in\left[s, t_{\mathrm{inf}}\right] \cap\left[t_{\mathrm{inf}}-r, t_{\mathrm{inf}}\right]$, then, according to (2.10) and (2.11), we obtain that

$$
\begin{aligned}
\left\|x\left(t_{\text {inf }}, \varphi\right)\right\|_{\infty} & =e^{-(\gamma / 2)\left(t_{\text {inf }}-s\right)}\|S(s, \varphi)\|_{\gamma, \infty} \\
& \geq e^{-(\gamma / 2)\left(t_{\text {inf }}-s\right)} e^{(\gamma / 2)(t-s)}\|x(t, \varphi)\|_{\infty} \\
& \geq e^{-\gamma r}\|x(t, \varphi)\|_{\infty}
\end{aligned}
$$

which proves (2.12) in this case.

Case (ii). If $t \in(-\infty, s] \cap\left[t_{\text {inf }}-r, t_{\text {inf }}\right]$, then, according to (2.10) and the definition of the norm $\|\cdot\|_{\gamma, \infty}$, we obtain that

$$
\begin{aligned}
\left\|x\left(t_{\text {inf }}, \varphi\right)\right\|_{\infty} & =e^{-(\gamma / 2)\left(t_{\text {inf }}-s\right)} \max _{\omega \in[-r, 0]} e^{\gamma \omega}\|S(s, \varphi)(\omega)\|_{\infty} \\
& =\max _{\omega \in[-r, 0]} e^{-(\gamma / 2)\left(t_{\text {inf }}-s-2 \omega\right)}\|x(s+\omega, \varphi)\|_{\infty} \\
& \geq e^{-(\gamma / 2)\left(t_{\text {inf }}+s-2 t\right)}\|x(t, \varphi)\|_{\infty} \\
& \geq e^{-\gamma r}\|x(t, \varphi)\|_{\infty} .
\end{aligned}
$$

Hence, (2.12) is proved. To conclude the proof of this step, we estimate the norm $\|x(t, \varphi)\|_{\infty}$ for all $t$ in a neighborhood of $t_{\text {inf }}$ in order to show a contradiction to the assumption that the set $\mathcal{N}$ is not empty. To this end, we define the following set:

$$
I:=\left\{i \in\{1, \ldots, d\}:\left|x_{i}\left(t_{\text {inf }}, \varphi\right)\right|=\left\|x\left(t_{\text {inf }}, \varphi\right)\right\|_{\infty}\right\} .
$$


The continuity of the functions $t \mapsto x_{i}(t, \varphi)$ for $i=1, \ldots, d$ implies that there exists a neighborhood $\left(t_{\text {inf }}-\varepsilon, t_{\text {inf }}+\varepsilon\right)$ for some $\varepsilon>0$ such that

$$
\|x(t, \varphi)\|_{\infty}=\max _{i \in I}\left|x_{i}(t, \varphi)\right| \quad \forall t \in\left(t_{\text {inf }}-\varepsilon, t_{\text {inf }}+\varepsilon\right) .
$$

By virtue of (2.1), the derivative of the function $t \mapsto x_{i}^{2}(t, \varphi)$ is estimated as follows:

$$
\begin{aligned}
\left.\frac{1}{2} \frac{d}{d t} x_{i}^{2}(t, \varphi)\right|_{t=t_{\text {inf }}}= & x_{i}\left(t_{\text {inf }}, \varphi\right)\left[a_{i i}\left(t_{\text {inf }}\right) x_{i}\left(t_{\text {inf }}, \varphi\right)\right. \\
& \left.+\sum_{j=1, j \neq i}^{d} a_{i j}\left(t_{\text {inf }}\right) x_{j}\left(t_{\text {inf }}-\tau_{i j}, \varphi\right)\right] \\
\leq & a_{i i}\left(t_{\text {inf }}\right) x_{i}\left(t_{\text {inf }}, \varphi\right)^{2} \\
& +\left|x_{i}\left(t_{\text {inf }}, \varphi\right)\right| \sum_{j=1, j \neq i}^{d}\left|a_{i j}\left(t_{\text {inf }}\right)\right|\left\|x\left(t_{\text {inf }}-\tau_{i j}, \varphi\right)\right\|_{\infty}
\end{aligned}
$$

which together with (2.12) and the definition of $I$ implies that for all $i \in I$

$$
\left.\frac{1}{2} \frac{d}{d t} x_{i}(t, \varphi)^{2}\right|_{t=t_{\mathrm{inf}}} \leq x_{i}\left(t_{\mathrm{inf}}, \varphi\right)^{2}\left[a_{i i}\left(t_{\mathrm{inf}}\right)+e^{\gamma r} \sum_{j=1, j \neq i}^{d}\left|a_{i j}\left(t_{\mathrm{inf}}\right)\right|\right]
$$

Thus, from the row diagonal dominance (2.4) and bound (2.5), we derive that

$$
\left.\frac{1}{2} \frac{d}{d t} x_{i}(t, \varphi)^{2}\right|_{t=t_{\mathrm{inf}}} \leq\left[-\delta+\left(e^{\gamma r}-1\right) M\right] x_{i}^{2}\left(t_{\mathrm{inf}}, \varphi\right) .
$$

Using (2.6), we obtain that

$$
\left.\frac{1}{2} \frac{d}{d t} x_{i}^{2}(t, \varphi)\right|_{t=t_{\text {inf }}}<-\frac{\gamma}{2} x_{i}^{2}\left(t_{\mathrm{inf}}, \varphi\right)
$$

which yields that there exists a neighborhood $\left(t_{\text {inf }}, t_{\text {inf }}+\varepsilon_{2}\right)$ for an $\varepsilon_{2}>0$ such that for $i \in I$

$$
\left|x_{i}(t, \varphi)\right| \leq e^{-(\gamma / 2)\left(t-t_{\text {inf }}\right)}\left|x_{i}\left(t_{\mathrm{inf}}, \varphi\right)\right| \quad \forall t \in\left(t_{\mathrm{inf}}, t_{\mathrm{inf}}+\varepsilon_{2}\right)
$$

Thus, for all $t \in\left(t_{\text {inf }}, t_{\text {inf }}+\varepsilon\right)$ with $\varepsilon:=\min \left\{\varepsilon_{1}, \varepsilon_{2}\right\}$, we have

$$
\|x(t, \varphi)\|_{\infty} \leq e^{-(\gamma / 2)\left(t-t_{\text {inf }}\right)}\left\|x\left(t_{\text {inf }}, \varphi\right)\right\|_{\infty}
$$


which together with (2.10) implies that

$$
\|x(t, \varphi)\|_{\infty} \leq e^{-(\gamma / 2)(t-s)}\|S(s, \varphi)\|_{\gamma, \infty}
$$

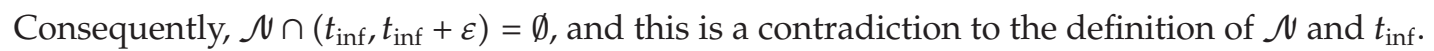
Thus, (2.8) is proved.

Step 2. Using (2.8) from Step 1, we show (2.7) by considering two cases: (i) $t \in[s+$ $r, T] \cap[s, T]$ and (ii) $t \in[s, s+r] \cap[s, T]$.

Case (i). If $t \in[s+r, T] \cap[s, T]$, then by virtue of (2.8) we have

$$
\begin{aligned}
\|S(t, \varphi)\|_{\gamma, \infty} & =\sup _{\omega \in[-r, 0]} e^{\gamma \omega}\|x(t+\omega, \varphi)\|_{\infty} \\
& \leq \sup _{\omega \in[-r, 0]} e^{\gamma \omega} e^{-(\gamma / 2)(t+\omega-s)}\|S(s, \varphi)\|_{\gamma, \infty} \\
& \leq e^{-(\gamma / 2)(t-s)}\|S(s, \varphi)\|_{\gamma, \infty^{\prime}}
\end{aligned}
$$

which proves (2.7) in this case.

Case (ii). If $t \in[s, s+r] \cap[s, T]$, then we have

$$
\begin{array}{r}
\|S(t, \varphi)\|_{\gamma, \infty}=\max \left\{\sup _{\omega \in[-(t-s), 0]} e^{\gamma \omega}\|x(t+\omega, \varphi)\|_{\infty}\right. \\
\left.\sup _{\omega \in[-r,-(t-s)]} e^{\gamma \omega}\|x(t+\omega, \varphi)\|_{\infty}\right\} .
\end{array}
$$

Hence, using (2.8), we obtain that

$$
\begin{gathered}
\|S(t, \varphi)\|_{\gamma, \infty} \leq \max \left\{\sup _{\omega \in[-(t-s), 0]} e^{(\gamma / 2) \omega} e^{-(\gamma / 2)(t-s)}\|S(s, \varphi)\|_{\gamma, \infty}\right. \\
\left.e^{-\gamma(t-s)}\|S(s, \varphi)\|_{\gamma, \infty}\right\} .
\end{gathered}
$$

Thus, (2.7) is proved, and the proof is complete.

\section{Finite-Time Attractivity of Lotka-Voltera Systems}

Consider a Lotka-Voltera system of the following form:

$$
\dot{x}_{i}(t)=x_{i}(t)\left(r_{i}+\sum_{j=1}^{d} a_{i j} x_{j}\left(t-r_{i j}\right)\right), \quad i=1, \ldots, d
$$


where $r_{i j} \geq 0$ and $r_{i i}=0$ for all $i=1, \ldots, d$. Suppose that $A=\left(a_{i j}\right)_{i, j=1, \ldots, d}$ is row diagonal dominant with $a_{i i}<0$ for all $i=1, \ldots, d$; that is, there exists $\delta>0$ such that

$$
\left|a_{i i}\right| \geq \sum_{j=1, j \neq i}^{d}\left|a_{i j}\right|+\delta .
$$

We assume in the following that there exists a positive vector $x^{*} \in \mathbb{R}_{+}^{d}$ such that

$$
\mathbf{r}+A x^{*}=0 \quad \text { where } \mathbf{r}=\left(r_{1}, \ldots, r_{d}\right)^{T}
$$

To shorten the notation, the function $t \mapsto x^{*}$ for all $t \in[-r, 0]$ is also denoted by $x^{*}$. The function $x^{*}$ is a fixed point of the evolution operator $S(t, \cdot)$ generated by $(3.1)$, that is, $S\left(t, x^{*}\right)=$ $x^{*}$ for all $t$. For system (3.1), the result in [13, Theorem 1] showed that the equilibrium $x^{*}$ is exponentially attractive on the positive real line $\mathbb{R}_{+}$; that is, there exist positive constants $K, \alpha$ and $\eta$ such that

$$
\left\|S(t, \varphi)-x^{*}\right\|_{0, \infty} \leq K e^{-\alpha t}\left\|\varphi-x^{*}\right\| \quad \forall \varphi \in B_{\eta}\left(x^{*}\right) .
$$

However, the constant $K$ is usually greater than 1 . Using the result developed in the preceding section, we show in the next theorem that the constant $K$ in (3.4) can be chosen to be equal to 1 on the state space $\mathcal{C}$ with norm $\|\cdot\|_{\gamma, \infty}$ for some $\gamma \geq 0$. As a consequence, the equilibrium solution of system (3.1) is finite-time attractive with respect to these norms.

Theorem 3.1 (finite-time attractive equilibrium of Lotka-Voltera equations). Consider (3.1) on an arbitrary finite-time interval $[0, T]$ satisfying (3.2) and (3.3). Then, there exists a positive weight factor $\gamma^{*}$ such that for all $\gamma \in\left[0, \gamma^{*}\right]$ the positive equilibrium $x^{*}$ is finite-time attractive on $[0, T]$ with respect to the norm $\|\cdot\|_{\gamma, \infty}$.

Proof. The proof is divided into three steps.

Step 1. Construction of the weight factor $\gamma^{*}$. Due to compactness of $[0, T]$ and continuity of solutions of (3.1), there exists $\eta^{*}>0$ such that

$$
\left\|S(t, \varphi)-x^{*}\right\|_{0, \infty} \leq \frac{\min _{i=1, \ldots, d} x_{i}^{*}}{2} \text { for } t \in[0, T], \varphi \in B_{\eta^{*}}\left(x^{*}\right) .
$$

Then, we have

$$
\left|x_{i}(t, \varphi)-x_{i}^{*}\right| \leq\left\|S(t, \varphi)-x^{*}\right\|_{0, \infty} \leq \frac{\min _{i=1, \ldots, d} x_{i}^{*}}{2}
$$

which implies that for all $t \in[0, T]$ and $\varphi \in B_{\eta^{*}}\left(x^{*}\right)$ we have

$$
\frac{\min _{i=1, \ldots, d} x_{i}^{*}}{2} \leq\left|x_{i}(t, \varphi)\right| \leq \frac{\min _{i=1, \ldots, d} x_{i}^{*}}{2}+\max _{i=1, \ldots, d} x_{i}^{*} .
$$


Define

$$
\delta^{*}:=\frac{\min _{i=1, \ldots, d} x_{i}^{*}}{2} \delta, \quad M:=\left(\frac{\min _{i=1, \ldots, d} x_{i}^{*}}{2}+\max _{i=1, \ldots, d} x_{i}^{*}\right) \max _{i=1, \ldots, d} \sum_{j=1, j \neq i}^{d}\left|a_{i j}\right| .
$$

Now let $\gamma^{*}>0$ be the solution of the following equation:

$$
\frac{r^{*}}{2}+\left(e^{r^{*} r}-1\right) M=\delta^{*}
$$

Step 2. In this step, we show that $y(t):=x(t, \varphi)-x^{*}$ is the solution of the delay equation

$$
\dot{y}_{i}(t)=a_{i i}(t) y_{i}(t)+\sum_{j=1, j \neq i}^{d} a_{i j}(t) y_{j}\left(t-r_{i j}\right) \quad \text { for } i=1, \ldots, d
$$

with the initial condition $y(s)=\varphi(s)-x^{*}$ for $s \in[-r, 0]$, where

$$
a_{i j}(t):=a_{i j} x_{i}(t, \varphi) \text { for } i, j=1, \ldots, d
$$

Indeed, we have

$$
\begin{aligned}
\dot{y}_{i}(t) & =x_{i}(t, \varphi)\left[r_{i}+\sum_{j=1}^{d} a_{i j} x_{j}\left(t-r_{i j}, \varphi\right)\right] \\
& =x_{i}(t, \varphi)\left[r_{i}+\sum_{j=1}^{d} a_{i j} x_{j}^{*}+\sum_{j=1}^{d} a_{i j} y_{j}\left(t-r_{i j}\right)\right],
\end{aligned}
$$

which together with the fact that $\mathbf{r}+A x^{*}=0$ implies that $y(t)$ is a solution of (3.10). Furthermore, we have

$$
S^{*}\left(t, \varphi-x^{*}\right)=S(t, \varphi)-x^{*} \quad \forall t \in[0, T]
$$

where $S^{*}$ denotes the evolution operator generated by (3.10).

Step 3. In this step, we show that for all $\gamma \in\left[0, \gamma^{*}\right]$ and $\varphi \in B_{\eta^{*}}\left(x^{*}\right)$ we have

$$
\left\|S(t, \varphi)-x^{*}\right\|_{\gamma, \infty} \leq e^{-\gamma(t-s) / 2}\left\|S(s, \varphi)-x^{*}\right\|_{\gamma, \infty} \quad \forall 0 \leq s \leq t \leq T
$$


Choose and fix $\varphi \in B_{\eta^{*}}\left(x^{*}\right)$, and we show that (3.10) fulfills all assumptions of Theorem 2.1. Indeed, using (3.7) and the definition of $M$, we obtain the following upper bound:

$$
\begin{aligned}
\max _{t \in[0, T], i=1, \ldots, d} \sum_{j=1, j \neq i}^{d}\left|a_{i j}(t)\right| & \leq\left(\frac{\min _{i=1, \ldots, d} x_{i}^{*}}{2}+\max _{i=1, \ldots, d} x_{i}^{*}\right) \max _{i=1, \ldots, d} \sum_{j=1, j \neq i}^{d}\left|a_{i j}\right| \\
& \leq M .
\end{aligned}
$$

Combining (3.2) and (3.7), we also get that

$$
\begin{aligned}
\left|a_{i i}(t)\right|-\sum_{j=1, j \neq i}^{d}\left|a_{i j}(t)\right| & =\left|x_{i}(t, \varphi)\right|\left(\left|a_{i i}\right|-\sum_{j=1, j \neq i}^{d}\left|a_{i j}\right|\right) \\
& \geq \frac{\min _{i=1, \ldots, d} x_{i}^{*}}{2} \delta=\delta^{*} .
\end{aligned}
$$

Therefore, system (3.10) fulfills all assumptions of Theorem 2.1. Then, the zero solution of system (3.10) is finite-time attractive with respect to the norm $\|\cdot\|_{\gamma, \infty}$ for all $\gamma \in\left[0, \gamma^{*}\right]$, that is,

$$
\left\|S^{*}(t, \psi)\right\|_{\gamma, \infty} \leq e^{-\gamma(t-s) / 2}\left\|S^{*}(s, \psi)\right\|_{\gamma, \infty} \quad \forall 0 \leq s \leq t \leq T, \psi \in \mathrm{C}
$$

In particular, substituting $\psi=\varphi-x^{*}$ we get that

$$
\left\|S^{*}\left(t, \varphi-x^{*}\right)\right\|_{\gamma, \infty} \leq e^{-(\gamma(t-s)) / 2}\left\|S^{*}\left(s, \varphi-x^{*}\right)\right\|_{\gamma, \infty} \quad \forall 0 \leq s \leq t \leq T
$$

which together with (3.13) implies (3.14) and the proof is complete.

In the rest of the paper, we discuss a planar Lotka-Voltera system, for which we can explicitly compute its equilibrium. Consequently, applying Theorem 3.1 yields a sufficient condition for finite-time attractivity of this equilibrium.

Example 3.2. Consider a planar Lotka-Voltera of the following form:

$$
\begin{aligned}
& \dot{x}_{1}(t)=x_{1}(t)\left(r_{1}+a_{11} x_{1}(t)+a_{12} x_{2}\left(t-\tau_{12}\right)\right), \\
& \dot{x}_{2}(t)=x_{2}(t)\left(r_{2}+a_{21} x_{1}\left(t-\tau_{21}\right)+a_{22} x_{2}(t)\right),
\end{aligned}
$$

where $r_{1}, r_{2}, \tau_{12}, \tau_{21}>0$ and the coefficients $a_{i j}$ for $i, j=1,2$ satisfy the following inequalities for some $\varepsilon>0$ :

$$
a_{11}, a_{22}<0, \quad\left|a_{11}\right| \geq\left|a_{12}\right|+\varepsilon, \quad\left|a_{22}\right| \geq\left|a_{21}\right|+\varepsilon .
$$


Additionally, we assume that the equilibrium point $x^{*}=\left(x_{1}^{*}, x_{2}^{*}\right)$ is positive, where

$$
x_{1}^{*}=\frac{r_{2} a_{12}-r_{1} a_{22}}{a_{11} a_{22}-a_{12} a_{21}}, \quad x_{2}^{*}=\frac{r_{1} a_{21}-r_{2} a_{11}}{a_{11} a_{22}-a_{12} a_{21}} .
$$

According to Theorem 3.1, for any finite-time interval $[0, T]$, there exists a positive weight factor $\gamma^{*}$ such that for all $\gamma \in\left[0, \gamma^{*}\right]$ the positive equilibrium $x^{*}$ is finite-time attractive on $[0, T]$ with respect to the norm $\|\cdot\|_{\gamma, \infty}$.

\section{References}

[1] A. Berger, D. T. Son, and S. Siegmund, "Nonautonomous finite-time dynamics," Discrete and Continuous Dynamical Systems. Series B, vol. 9, no. 3-4, pp. 463-492, 2008.

[2] G. Haller, "A variational theory of hyperbolic Lagrangian Coherent Structures," Physica D, vol. 240, no. 7, pp. 574-598, 2011.

[3] S. C. Shadden, F. Lekien, and J. E. Marsden, "Definition and properties of Lagrangian coherent structures from finite-time Lyapunov exponents in two-dimensional aperiodic flows," Physica D, vol. 212, no. 3-4, pp. 271-304, 2005.

[4] A. Berger, T. S. Doan, and S. Siegmund, "A definition of spectrum for differential equations on finite time," Journal of Differential Equations, vol. 246, no. 3, pp. 1098-1118, 2009.

[5] M. Branicki and S. Wiggins, "Finite-time Lagrangian transport analysis: stable and unstable manifolds of hyperbolic trajectories and finite-time Lyapunov exponents," Nonlinear Processes in Geophysics, vol. 17, no. 1, pp. 1-36, 2010.

[6] T. S. Doan, K. Palmer, and S. Siegmund, "Transient spectral theory, stable and unstable cones and Gershgorin's theorem for finite-time differential equations," Journal of Differential Equations, vol. 250, no. 11, pp. 4177-4199, 2011.

[7] T. S. Doan, D. Karrasch, T. Y. Nguyen, and S. Siegmund, "A unified approach to finite-time hyperbolicity which extends finite-time Lyapunov exponents," Journal of Differential Equations, vol. 252, no. 10, pp. 5535-5554, 2012.

[8] L. H. Duc and S. Siegmund, "Hyperbolicity and invariant manifolds for planar nonautonomous systems on finite time intervals," International Journal of Bifurcation and Chaos in Applied Sciences and Engineering, vol. 18, no. 3, pp. 641-674, 2008.

[9] M. Rasmussen, "Finite-time attractivity and bifurcation for nonautonomous differential equations," Differential Equations and Dynamical Systems, vol. 18, no. 1-2, pp. 57-78, 2010.

[10] P. Giesl and M. Rasmussen, "Areas of attraction for nonautonomous differential equations on finite time intervals," Journal of Mathematical Analysis and Applications, vol. 390, no. 1, pp. 27-46, 2012.

[11] F. Amato, M. Ariola, M. Carbone, and C. Cosentino, "Finite-time control of linear systems: a survey," in Current Trends in Nonlinear Systems and Control, Systems \& Control: Foundations \& Applications, pp. 195-213, Birkhäuser, Boston, Mass, USA, 2006.

[12] P. Dorato, "An overview of finite-time stability," in Current Trends in Nonlinear Systems and Control, Systems \& Control: Foundations \& Applications, pp. 185-194, Birkhäuser, Boston, Mass, USA, 2006.

[13] J. Hofbauer and J. W.-H. So, "Diagonal dominance and harmless off-diagonal delays," Proceedings of the American Mathematical Society, vol. 128, no. 9, pp. 2675-2682, 2000.

[14] A. M. Fink, Almost Periodic Differential Equations, vol. 377 of Lecture Notes in Mathematics, Springer, Berlin, Germany, 1974. 


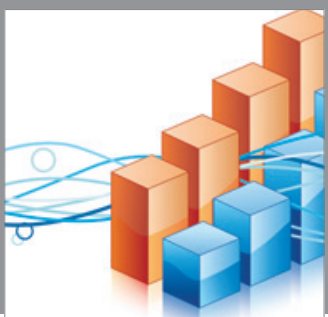

Advances in

Operations Research

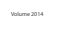

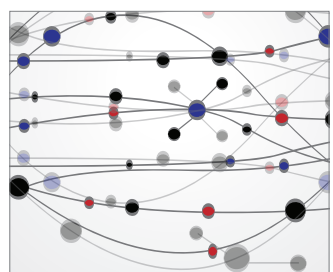

\section{The Scientific} World Journal
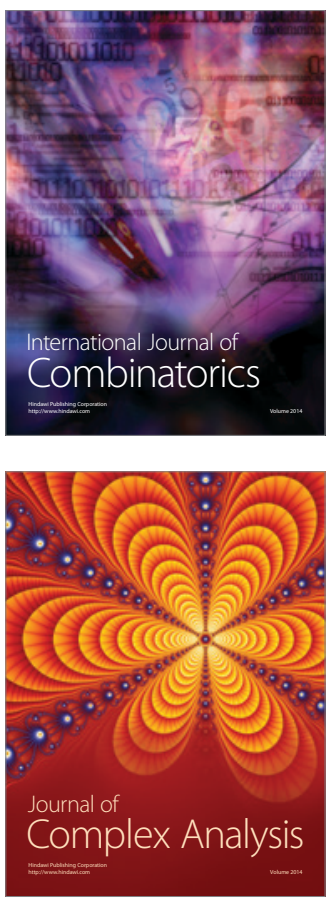

International Journal of

Mathematics and

Mathematical

Sciences
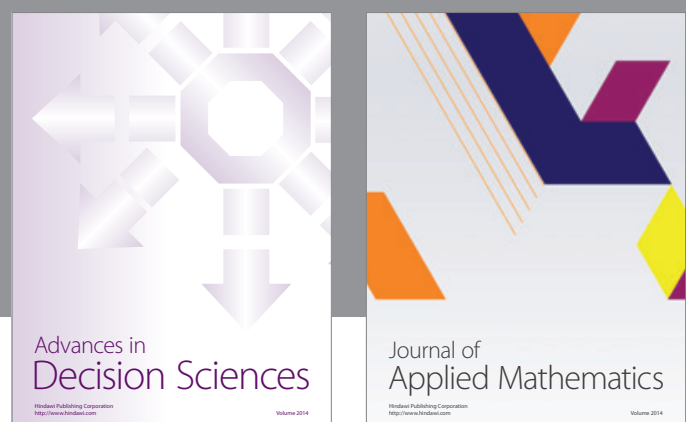

Journal of

Applied Mathematics
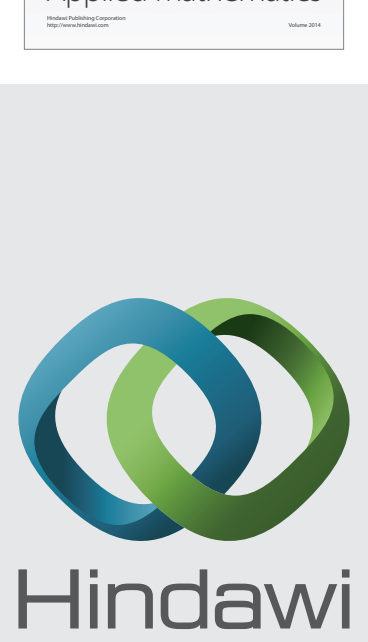

Submit your manuscripts at http://www.hindawi.com
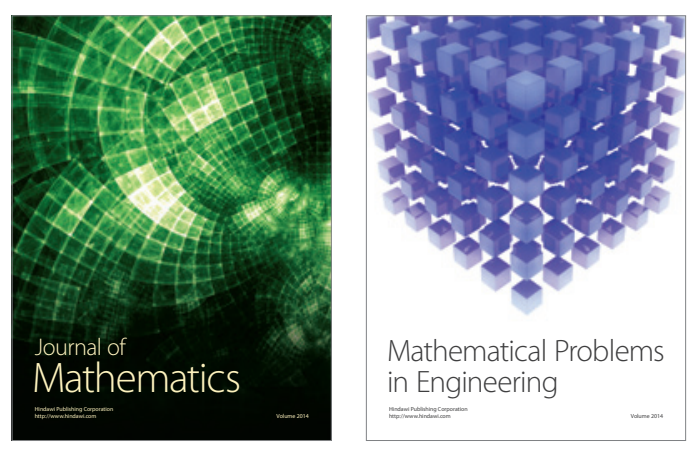

Mathematical Problems in Engineering
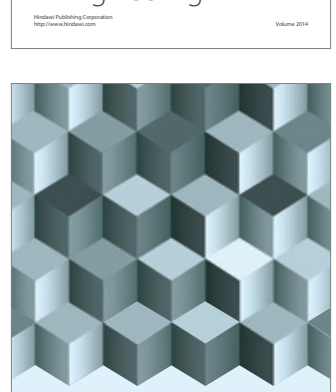

Journal of

Function Spaces
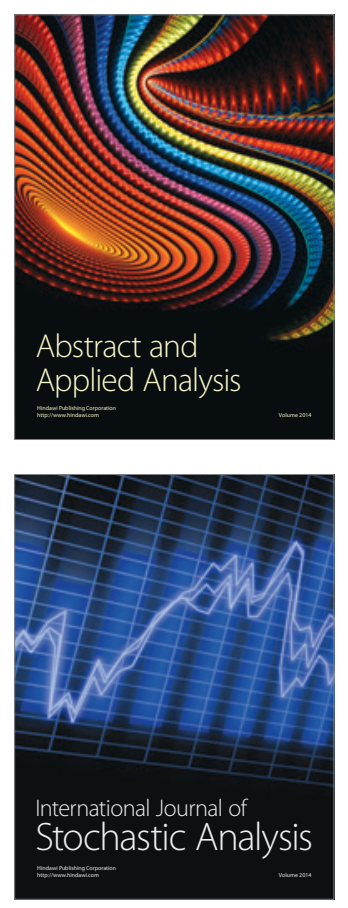

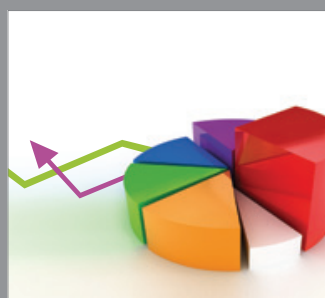

ournal of

Probability and Statistics

Promensencen
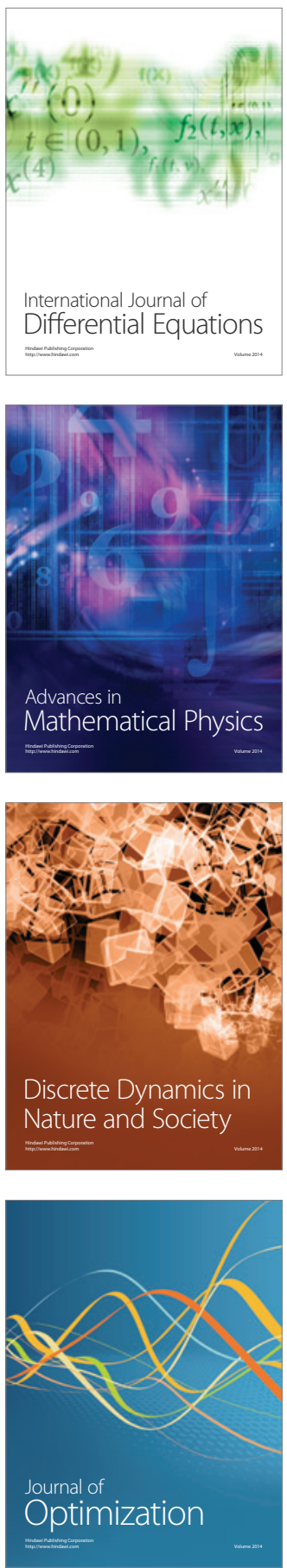